\title{
Study and Modeling of Human Biological Tissue Exposed to High Frequency Electromagnetic Waves
}

\author{
Anthony Bassesuka Sandoka Nzao \\ ISTA Kinshasa, Electrical Engineering, Kinshasa, Congo \\ Email: bass_sandoka@yahoo.fr
}

How to cite this paper: Nzao, A.B.S. (2021) Study and Modeling of Human Biological Tissue Exposed to High Frequency Electromagnetic Waves. Open Journal of Applied Sciences, 11, 1109-1121. https://doi.org/10.4236/ojapps.2021.1110083

Received: July 13, 2021

Accepted: October 22, 2021

Published: October 25, 2021

Copyright (c) 2021 by author(s) and Scientific Research Publishing Inc. This work is licensed under the Creative Commons Attribution International License (CC BY 4.0).

http://creativecommons.org/licenses/by/4.0/

Open Access

\begin{abstract}
The main objective of this proposed article is to provide explanations to justify the validity of the results of the studies of the interaction between the electromagnetic fields and the human body. It can also find direct applications in the characterization and modeling of the macroscopic electrical properties of the biological media for assessing the effects of fields induced by electromagnetic radiation sources in the human body to set up new standards on the Human exposure to electromagnetic fields. To do this, we have taken into account the different physical phenomena of propagation of a hyper-frequency electromagnetic plane wave and on the other hand, the experimental values in order to model the electrical behavior of human biological tissues based on an equivalent electronic circuit model composed of capacities, resistance and reel, which assimilates the biological tissues of the skin, grease, blood. This model using the characteristic impedance of the dielectric support makes it possible to evaluate the voltage induced by the electromagnetic waves of the hyper-frequencies in the studied biological system. The results of the simulations obtained from computer tools demonstrate that the hyper-frequency electromagnetic waves can result in an elevation of the electrical potential of the biological tissues. Despite this potential is a decreasing function of the penetration depth.
\end{abstract}

\section{Keywords}

Modeling, Equivalent Electronic Circuit, Biological Tissue, Microwave Electromagnetic Wave, Maxwell Equations

\section{Introduction}

The omnipresence of sources of electromagnetic fields in industrialized coun- 
tries means that people residing in these countries are exposed to them on a daily basis, both in their domestic and professional environment [1] [2]. However, it is in the professional environment that these sources are most common and also most intense [3]. The potential risks for people exposed to interactions between electromagnetic fields and the human body are real and therefore require protective measures [3]. Exposure to these sources may be voluntary in the case of medical diagnosis such as an NMR imager, hyperemia therapy, ablatherapy or involuntary mobile telephony, radio or television transmitters, household appliances, computer screens, televisions, security systems in shops and airports, high voltage lines, transformers, etc. [4]. Exposure to electromagnetic fields generates currents inside the body, the corresponding absorption of energy in the tissues results in an increase in temperature [5]. The health effects generated are mainly a function of the coupling mechanism, the nature of the fields and the duration of exposure [6]. These phenomena are all the more important as the intensity and/or the frequency of the signal are high [3] [7]. Apart from the effects observed on biological functions and on health, electromagnetic fields and waves also act on electronic devices [8]. Therefore, it is important during the construction of the latter to ensure that their operation does not interfere with that of other devices or is not itself a victim of other devices that are sources of fields. To avoid all forms of electromagnetic compatibility [9] [10] [11].

Several epidemiological and experimental studies have been conducted on this and they led most to establish biological effects that may mean risk or long-term health of a living being [12]. Theoretical studies have also made it possible to estimate doses of electromagnetic energy absorbed by animals and humans.

The effects of electromagnetic fields on a biological cell being the objective of this article, it seems essential to us to describe the biological cell and the molecular structure of the plasma membrane to underline the difficulties related to their electrical characterization [13]. This is how we have taken into account the analyses of some of the authors cited below to help us orient our work [1] [9] [12] [14] [15].

According to TBCarlos KONLACK and Roger TCHUIDJAN [2011] an evaluation of the power distribution induced by an electromagnetic wave in a spherical model of the brain allows us to say that [8], the quantity of energy received by the brain from the electromagnetic waves is very high compared to that which emerges from it by radiation, and that almost all of the energy received is transformed into heat.

According to D.V. Land [3], microwave thermography techniques have been used extensively in medical applications to monitor tissue temperature and detect the electromagnetic field in biological tissues. Since the temperature increases in the resulting tissue from the deposition of energy and is proportional to the square of the electric field in the tissue; the response to thermal radiation must have the same sample.

The various studies carried out on the subject have shown that the electrical impulses induced by microwave electromagnetic waves can cause cell death. The 
mechanisms leading to this destruction may differ depending on the characteristics of the pulse, the number as well as the frequency of repetition [16] [17].

According to K.H. Schoenbach, S. Xiao, R.P. Joshi, J.T. Camp, T. Heeren, J.F. Kolb and S.J. Beebe [2012] [18], the duration of the pulse is close to a nanosecond and the applied field is of the order of $\mathrm{MV} / \mathrm{cm}$. The death of illuminated cells is caused purely by modifications of the different electrical potentials of the different membranes. The study J.T. Camp, Y. Jing, J. Zhuang, J.F. Kolb, S.J. Beebe, J. Song, R.P. Joshi, S. Xiao and K.H. Schoenbach [2012] [18] show that, cell death is caused by electrical effects, but also by thermal effects.

A biological system irradiated by an electromagnetic wave is crossed by induced currents of significant density [18] [19] [20]. The use of high frequencies, of the order of tens of gigahertz and more, can cause non-thermal effects that are damaging to the health of an exposed biological system. This depends on the frequency, intensity of these waves and the duration of exposure to them.

Thus, the mathematical and numerical approach that we propose in our article is intended to be at the same time analytical, comparative and critical [12]. The aim is not only to analyze, but also to compare the various theories of specialists in the field in order to draw useful lessons for analyzing and evaluating the voltage induced by microwave waves in the various biological tissues studied.

Our work is aimed at complementing and reinforcing the veracity of some of the results already obtained. For our application, we will consider the particular case of skin, blood and muscle subjected to electromagnetic radiation from microwave frequencies.

\section{Development}

\subsection{Study Method}

To analyze the electrical behavior of biological tissue exposed in an electromagnetic environment of high frequency and predict possible consequences on health, we will proceed by a mathematical and numerical analysis based on the equivalent electronic circuit method, using the characteristic impedance of the media biological by involving bioelectrical parameters (capacitance, resistance and coil). This is a study based on solving Maxwell's equations that govern the behavior of an electromagnetic plane wave in the deferent models used. We will then proceed by modeling and simulating the voltage and current induced for biological media.

There is some need for approximation in this study. Biological systems are quite complex due to their geometric shape and the inhomogeneity of their internal constitution. This is how we consider a living, lossy, homogeneous and isotropic biological system in order to simplify the resolution of wave equations. Knowing that biological tissue is divided into two broad categories, tissue with high water content such as skin, blood and muscle and tissue with low water content such as fat and bone, the characteristics of which vary from a different way.

These approaches will allow us to make an analytical and theoretical approx- 
imation of this article as listed above.

\subsection{Brief Description of the Biological Cell}

Since the effects of electromagnetic fields in a biological cell are the focus of this article, we believe it is essential to describe the biological cell and the molecular structure of the plasma membrane. The biological cell is the structural and functional unit of all living things, it is characterized by its nucleus, cytoplasm and plasma membrane [21]. The cell plasma membrane plays an essential role in the life of the cell, it delimits the cell and separates the cytoplasm from the external environment. It surrounds the cytosol (i.e. the liquid phase in which the cytoplasmic organelles bathe) and forms a very thin protective layer made up of lipid and protein molecules. It thus presents a heterogeneous molecular structure allowing it to play a double role: the phospholipids which are the essential constituents of its basic material, make it insulating, while the protein molecules which are scattered, ensure the exchange between the cytoplasm and the environment extra cellular [22].

The cell is the basic structural and functional unit of all living things. The cells are very small and very complex in organization. Knowledge of their structure, chemical composition and functioning (physiology) is very critical in biology and biomedical science. Studies on cellular functionality and behavior have been widely applied in many clinical and biomedical applications, such as disease diagnosis and knowledge of their degree of evolution, drug development and cancer research [19] [23].

This is how knowledge of their component and their characteristic is important for the further development of our research and especially for the study and modeling of human biological tissue exposed to microwave electromagnetic waves.

\subsection{Brief Description of the Biological Cell}

In the case of biological media, the phenomena of energy absorption in tissues can be relatively complex and depend on many factors. They are primarily linked to the type of coupling between the emission source and the biological medium. Galvanic coupling corresponds to the case of physical contact between the source and the medium. This contact causes an ohmic-type current to flow through the person's body. Depending on the intensity and frequency of the contact current, the consequences can result in tissue heating or even a burn.

Radiated coupling comprises three fundamental mechanisms by which electric and/or magnetic fields, of variable frequency over time, interact with biological media.

- Coupling with low-frequency electric fields: the external electric fields induce, on the surface of the exposed body, a surface charge which causes, inside the body, the appearance of currents whose distribution depends on the conditions of exposure, the size and shape of the body as well as the position of the body vis-à-vis the field. 
- Coupling with low frequency magnetic fields: the physical interaction between magnetic fields of variable frequency and the human body creates induced electric fields and causes the circulation of electric currents. The magnitude of the fields and the density of the induced current are proportional to the strength and frequency of the magnetic field $\mathrm{B}$, the radius of the current loop in the body, and the electrical conductivity of the exposed tissues.

- It should be noted that exposure of the human body to low-frequency electric or magnetic fields generally results in only negligible energy absorption and no measurable temperature rise. On the other hand, exposure to electromagnetic fields with a frequency greater than $100 \mathrm{kHz}$ can cause energy absorption and a significant rise in temperature. In general, exposure to electromagnetic fields results in a highly inhomogeneous deposition and distribution of energy within the body which must be assessed by dosimetry. Finally, it remains to underline the indirect consequences caused by exposure to electromagnetic fields of people with active medical implants (cardiac pacemaker, cardiac defibrillator, insulin pump, etc.) which result in malfunctions of the implanted equipment.

These different phenomena can be analyzed mathematically in detail from Maxwell's equations by defining each parameter and its role, some details of which are not given.

\section{Biological Tissue Modeling}

\subsection{The Human Body and Sources of Electromagnetic Fields}

Humans live in an electromagnetic environment created by many field sources. These sources can have very different characteristics of frequency, voltage levels, current or power [20]. They come in a variety of forms such as the power line, telecommunication relays, induction welding tools, cell phones or household electrical devices (hair dryer, induction hob, microwave oven) [24]. In many situations, the presence of the human body in the radiation area of the source does not affect the power emitted. If the source of the electromagnetic field is known (following in situ measurements or a preliminary calculation), it is possible not to explicitly model the source but only the human body [17]. This feature is often taken advantage of in models to limit their complexity [25].

The difficulties in modeling human exposure to electromagnetic fields are due to the particular electrical and geometric properties of the body and the variety of radiation sources.

The response of a cell in the presence of an electric field depends on two parameters which are the relative dielectric permittivity which reflects the capacity to polarize a material by accumulation of charges and the electrical conductivity which reflects the capacity to pass an electric current with minimal losses. The fundamental concepts of dielectric phenomena in biological media and their interpretation of interactions at the cellular level are well established [26] [27] [28]. Based on the work of Prof. Schwan [29] [30] and Foster [31], the dielectric 
properties of cells depend on frequency and exhibit relaxation and resonance phenomena, which are a function of different polarizations. The relaxations are named $\alpha, \beta$ and $\gamma$ and are more often referred to by the term dispersion because the resulting dielectric absorption is observable over a wide range of frequencies [25] [27] [28] [29] [31].

Different empirical models can be used to approximate the frequency variations of the electrical properties of cells. Electrical modeling of cells was first proposed by R. Höber in the 1910s, who studied the evolution of the resistivity of a blood sample at low and high frequencies. As a result of these early observations, several electrical models of the cell were constructed in the mid-twenty-second century, such as the Fricke, Debye and Cole-Cole model. These models are still widely used today in studies of the electrical behavior of biological media.

In this paper, the modeling of human biological tissue is based on the equivalent electronic circuit model shown below, similar to any dielectric medium and able to model all related properties [32] [33] [34] [35].

In Figure 1, $R$ : represents resistance in Ohm per meter $\left[\mathrm{W} \cdot \mathrm{m}^{-1}\right], G$ : conductance in $\mathrm{Oh} \cdot \mathrm{m}^{-1}$ per meter: $L$ : inductance in micro Henry per meter $\left[\mathrm{mH} \cdot \mathrm{m}^{-1}\right]$, and $C$ : Capacitance in Pico Faraday per meter $\left[\mathrm{pF} \cdot \mathrm{m}^{-1}\right]$ [36].

Table 1 assembles the values of the parameters $R, G, L$ and $C$ modeled for different media. These are the electrical parameters, used in our model that make up the electronic circuit as shown in Figure 1, which is similar to any biological dielectric medium [30] [36] [37].

\subsection{Reliability of the Model}

From a reliability point of view, this equivalent electronic circuit model reproduces the electrical behavior of a biological tissue with maximum precision, without taking into account its internal structure. It also reduces simulation time and gives the possibility of simulating complex systems like the one being the subject of our study [27].

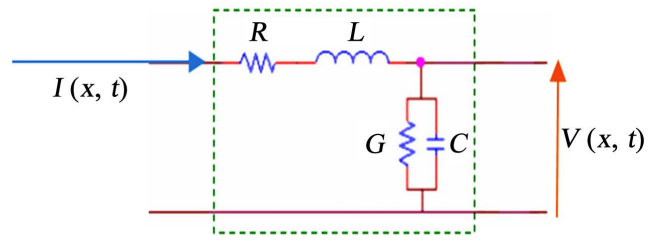

Figure 1. Circuit electronic equivalent of a biological tissue.

Table 1. Values of bioelectric parameters $R, G, L$ and $C$.

\begin{tabular}{cccc}
\hline Bioelectric Paramters & blood & Muscle & Skin \\
\hline$R$ & 0 & 0 & 0 \\
$G$ & 2.623 & 4.037 & 3.533 \\
$L$ & 1.257 & 1.257 & 1.257 \\
$C$ & 6.72 & 6.552 & 3.542 \\
\hline
\end{tabular}




\subsection{Voltage and Current Induced in the Biological Tissue by the Progation of Microwave Waves}

The model of Figure 1 above makes it possible to indirectly obtain the mathematical expressions of the voltage $V(x, t)$ and of the current $I(x, t)$ thus induced from the propagation impedance of the wave by involving the model electrical parameters ( $R, L, C$ and $G$ ) [8] [38] [39] [40].

In order to define the different equations to obtain an adequate analytical model (see Equations (1)-(7)), Kirchooff's laws (mesh and node laws) have been used, see Figure 1. Thus, the following mathematical expressions are deduced:

$$
\begin{gathered}
V(x, t)=(R+j L \omega) \cdot I(x, t) \\
I(x, t)=(G+j C \omega) \cdot V(x, t) \\
V(x, t)=V_{0} \cdot \exp (-\alpha x) \cdot \cos (\omega t-\beta x) \\
I(x, t)=\left(I_{0} / Z_{0}\right) \cdot \exp (-\alpha x) \cdot \cos (\omega t-\beta x) \\
\gamma=\sqrt{(R+j L \omega) \cdot(G+j C \omega)} \\
\gamma=\alpha+j \beta \\
Z_{0}=\sqrt{(R+j L \omega) /(G+j C \omega)}
\end{gathered}
$$

These equations are used to simulate the electrical behavior of the biological tissues studied.

\section{Electrical Behavior Simulation}

\subsection{Calculation of Bioelectronic Parameters}

By using computer tools such as Matlab and others for the evaluation of bioelectronic parameters, we obtained the following results: $a=79 ; b=81 ; R_{e}\left(Z_{0}\right)=167$ Ohm; $\operatorname{Im}\left(Z_{0}\right)=8 \mathrm{Ohm} ; R_{e}(g)=79 ; \operatorname{Im}(g)=4$.

\subsection{Results}

Considering the frequency of 3.2 Gigas Hertz [Ghz] of the propagation of electromagnetic waves and the depth of penetration of electromagnetic waves in a biological medium varies from 0 to 30 millimeters. The results of the simulation of the voltage induced by electromagnetic waves in the different biological media are in Figures 2-5 below.

Figure 6 below presents the results obtained experimentally in the work of M. Mimi and D. V. Land [1991] [41].

\section{Discussion}

By simulating different biological tissues (blood, muscle and skin) we see that, the voltage induced by the electromagnetic wave of the microwaves increases, the thickness decreases as a function of the depth of penetration see Figure 2.

It has also been observed that the more the muscle thickness increases, the induced tension decreases. See Figure 3 and Figure 4 except that, the numerical values are different. 


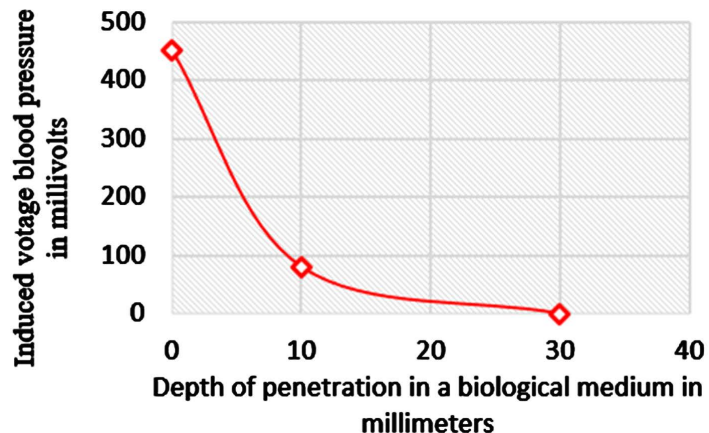

Figure 2. Evolution of the reflected voltage as a function of the depth of penetration into the blood.

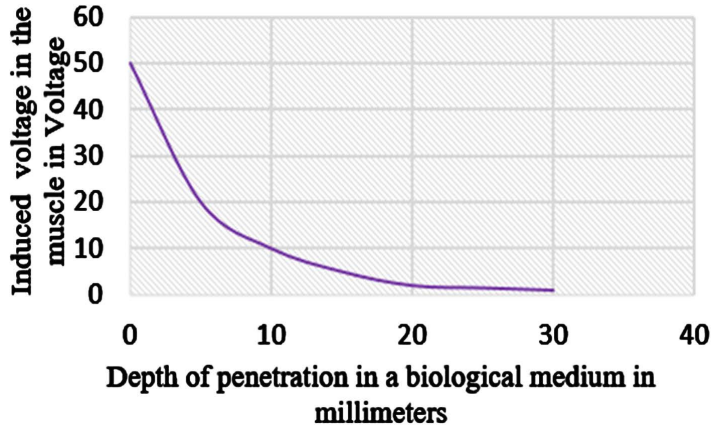

Figure 3. Evolution of the reflected voltage as a function of the depth of penetration into the skin.

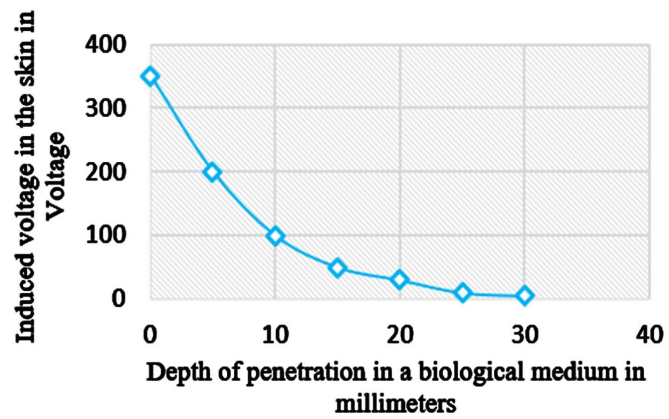

Figure 4. Evolution of the reflected tension as a function of the depth of penetration into the muscle.

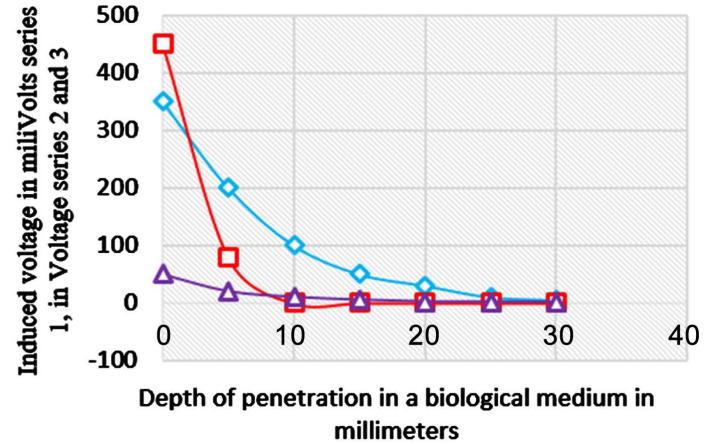

Figure 5. Superposition curve of the evolution of the reflected voltages as a function of the depth of penetration into the biological tissues under study. 


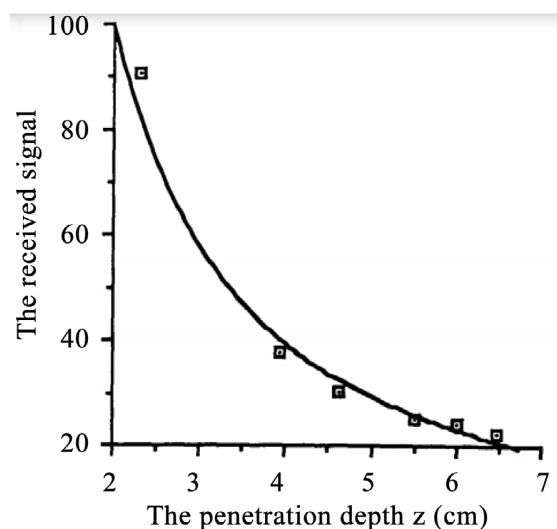

(a)

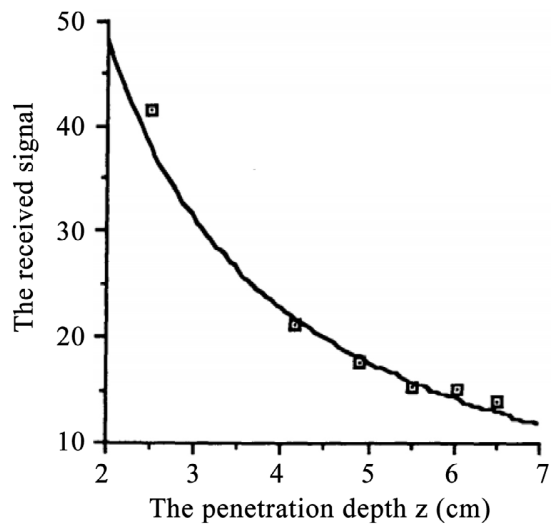

(c)

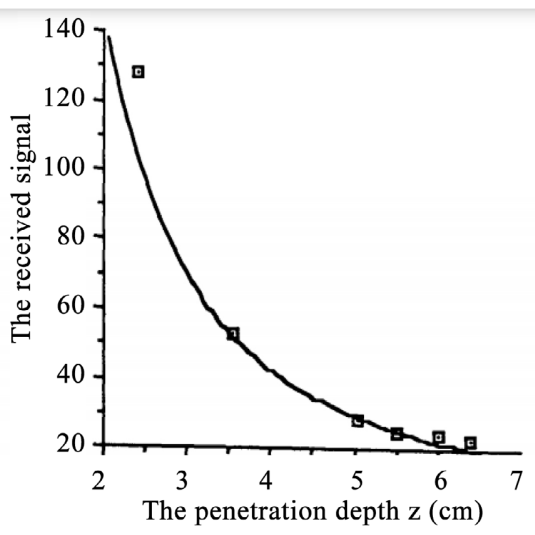

(b)

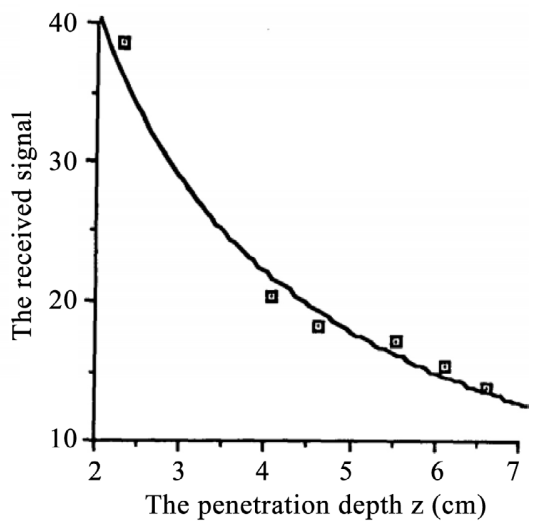

(d)

Figure 6. The penetration depth for muscle phantom material at $3.2 \mathrm{GHz}$ of crossed-pair antenna.

These results can be compared with those obtained experimentally in the work of M. Mimi and D.V. Land [1991] in Figure 6 above. We note from the above that the electrical impulses of microwave electromagnetic waves cause an increase in potential in the different biological media studied. This potential is a decreasing function of the penetration depth and it is very high at the end of the biological tissue.

However, the use of high frequencies, of the order of tens of gigahertz, can cause non-thermal effects which are harmful to health.

The various studies carried out on the subject have shown that the electrical impulses induced by microwave electromagnetic waves can cause cell death. The mechanisms leading to this destruction may differ depending on the characteristics of the pulse, the number as well as the frequency of repetition.

\section{Conclusions}

In this article we have chosen the modeling approach based on the equivalent electronic circuit of a human biological tissue, taking into account on the one hand the physical phenomena of the propagation of a microwave electromagnetic plane wave and on the other hand the values experiments in order to si- 
mulate the electrical behavior of human biological tissues exposed in a microwave electromagnetic environment.

The proposed work aims mainly to provide arguments to justify the relevance of the results of studies of the interaction between electromagnetic fields and the human body. It can also find direct applications in the precise characterization of biological media and in the establishment of new standards on human exposure to electromagnetic fields.

We have tried to take advantage of the many advantages of this method, namely the reduction of simulation time, the possibility of simulating complex systems like the one being the subject of our study.

The simulation result obtained in this article approaches that obtained experimentally in the work of M. Mimi and D.V. Land. We note from the above that the induced tension is important in the different biological media studied in this article, and is a decreasing function of the depth of penetration as the thickness of biological tissue is reduced.

As a result, we therefore deemed it useful that the modeling based on the equivalent electronic circuit of a human biological tissue analyzed from Kirchhoff's laws is more suitable for the study of a system as complicated and disparate as a tissue complex biological.

Of all the analyzes and modeling, Maxwell's equations can also be used in detail for modeling human biological tissue exposed to microwave electromagnetic waves, taking into account all of these parameters.

\section{Conflicts of Interest}

The author declares no conflicts of interest regarding the publication of this paper.

\section{References}

[1] Zoltowski, P. (1998) On the Electrical Capacitance of Interfaces Exhibiting Constant Phase Element Behavior. Journal of Electroanalytical Chemistry, 443, 149-154. https://doi.org/10.1016/S0022-0728(97)00490-7

[2] Wanichapichart, P., Bunthawin, S., Kaewpaiboon, A. and Kanchanapoom, K. (2002) Determination of Cell Dielectric Properties Using Dielectrophoretic Technique. Science Asia, 28, 113-119. https://doi.org/10.2306/scienceasia1513-1874.2002.28.113

[3] Mimi, M. and Land, D.V. (1991) Measurement of Non-Resonant Disturbance of Antenna Electromagnetic Field Configurations for Biomedical Applications. The Journal of Photographic Science, 39, 161-163. https://doi.org/10.1080/00223638.1991.11737141

[4] Simicevic, N. and Haynie, D.T. (2005) FDTD Simulation of Exposure of Biological Material to Electro-Magnetic Nanopulses. Physics in Medicine and Biology, 50, 347-360. https://doi.org/10.1088/0031-9155/50/2/012

[5] Wang, Y., Schimpf, P.H., Haynor, D.R. and Kim, Y. (1998) Geometric Effects on Resistivity Measurements with Four-Electrodeprobes in Isotropic and Anisotropic Tissues. IEEE Transactions on Biomedical Engineering, 45, 877-884. https://doi.org/10.1109/10.686795 
[6] Stuchly, M.A. and Dawson, T.W. (2000) Interaction of Low-Frequency Electric and Magnetic Fields with the Human Body. Proceedings of the IEEE, 88, 643-664. https://doi.org/10.1109/5.849161

[7] Schoenbach, K.H., Xiao, S., Joshi, R.P., Camp, J.T., Heeren, T., Kolb, J.F. and Beebe, S.J. (2008) The Effect of Intense Subnanosecond Electrical Pulses on Biological Cells. IEEE Transaction on Plasma Science, 36, 414-422.

https://doi.org/10.1109/TPS.2008.918786

[8] Reivonen, S., Keikko, T., Isokorpi, J. and Korpinen, L. (1999) Internal Currents in a Human Body with Spheroidal Model in $400 \mathrm{kV}$ Switching Substation. 11 th International Symposium on High-Voltage Engineering, London, 23-27 August 1999, 4 https://doi.org/10.1049/cp:19990588

[9] Miranda, P.C., Hallett, M. and Basser, P.J. (2003) The Electric Field Induced in the Brain by Magnetic Stimulation: A 3-D Finite Element Analysis of the Effect of Tissue Heterogeneity and Anisotropy. IEEE Transactions on Biomedical Engineering, 50, 1074-1085. https://doi.org/10.1109/TBME.2003.816079

[10] Raicu, V., Kitagawa, N. and Irimajiri, A. (2000) A Quantitative Approach to the Dielectric Properties of the Skin. Physics in Medicine \& Biology, 45, L1-L4.

https://doi.org/10.1088/0031-9155/45/2/101

[11] Galeev, A.L. (2000) The Effects of Microwave Radiation from Mobile Telephones on Human and Animals. Neuroscience and Behavioral Physiology, 30, 187-194. https://doi.org/10.1007/BF02463157

[12] Matsumoto, T., Chiba, A., Hayashi, N. and Isaka, K. (1999) Effect of Competitor ELF Electric and Magnetic Fields on Induced Current Density in Biological Model in the Vicinity of the Ground. 11 th International Symposium on High-Voltage Engineering, London, 23-27 August 1999, 3. https://doi.org/10.1049/cp:19990587

[13] Yamazaki, K., Kawamoto, T., Fujinami, H. and Shigemitsu, T. (2001) Investigation of ELF Magnetically Induced Current Inside the Human Body: Development of Estimation Tools and Effect of Organ Conductivity. Electrical Engineering Journal, 134, 1-10. (In Japan). https://doi.org/10.1002/1520-6416(20010130)134:2\%3C1::AID-EEJ1\%3E3.0.CO;2-6

[14] Gurtovenko, A.A. and Anwar, J. (2007) Modulation of the Structure and Properties of Cell Membranes: Molecular Mechanism of Action of Dimethyl Sulfoxide. Journal of Physical Chemistry B, 111, 10453-10460. https://doi.org/10.1021/jp073113e

[15] Robillard, P.N. and Poussart, D. (1979) Spatial Resolution of Four Electrode Array. IEEE Transactions on Biomedical Engineering, 26, 465-470.

https://doi.org/10.1109/TBME.1979.326572

[16] Jaspard, F., Nadi, M. and Rouane, A. (2003) Dielectric Properties of Blood: An Investigation of Haematocrit Dependence. Physiological Measurement, 24, 137-147. https://doi.org/10.1088/0967-3334/24/1/310

[17] Jossinet, J. (1998) The Impedivity of Freshly Excised Human Breast Tissue. Physiological Measurement, 19, 61-75. https://doi.org/10.1088/0967-3334/19/1/006

[18] Camp, J.T., Jing, Y., Zhuang, J., Kolb, J.F., Beebe, S.J., Song, J., Joshi, R.P., Xiao, S. and Schoenbach, K.H. (2012) Cell Death Induced by Subnanosecond Pulsed Electric Fields at Elevated Temperatures. IEEE Transactions on Plasma Science, 40, 2334-2347. https://doi.org/10.1109/TPS.2012.2208202

[19] Wtorek, J., Bujnowski, A., Poli ski, A., Jozefiak, L. and Truyen, B. (2004) A Probe for Immittance Spectroscopy Based on the Parallel Electrode Technique. Physiological Measurement, 25, 1249-1260. https://doi.org/10.1088/0967-3334/25/5/014

[20] Scorretti, R. (2003) Numerical and Experimental Characterization of the LF Mag- 
netic Field Generated by Electrotechnical Systems with a View to Modeling the Currents Induced in the Human Body. PhD Thesis, Lyon Central School, Lyon.

[21] Purschke, M., Laubach, H.-J., Rox Anderson, R. and Manstein, D. (2010) Thermal Injury Causes DNA Damage and Lethality in Surrounding Unheated Cells: Heat Spectator Effect Active. Journal of Investigative Dermatology, 130, 86-92. https://doi.org/10.1038/jid.2009.205

[22] Fear, E.C. and Stuchly, M.A. (1998) Modeling of Assemblages of Biological Cells Exposed to Electric Fields. IEEE Transactions on Biomedical Engineering, 45, 1259-1271. https://doi.org/10.1109/10.720204

[23] Bowers, J.C. and Neinhaus, H.A. (1989) SPICE2 Computer Models for HEXFES, International Rectifier HEXFET Data Book, PP. A153-A160.

[24] Schharfetter, H. (1999) Structural Modeling for Non-Invasive Impedance-Based Diagnostic Methods. Habilitation Thesis, Faculty of Electrical Engineering, Technical University of Graz, United Kingdom.

[25] Jaspard, F. and Nadi, M. (2002) Dielectric Properties of Blood: An Investigation of Temperature Dependence. Physiological Measurement, 23, 547-554.

https://doi.org/10.1088/0967-3334/23/3/306

[26] Kyle, A.H., Chan, C.T.O. and Minchinton, A.I. (1999) Characterization of ThreeDimensional Tissue Cultures Using Electrical Impedance Spectroscopy. Biophysical Journal, 76, 2640-2648. https://doi.org/10.1016/S0006-3495(99)77416-3

[27] De Ménorval, M.-A., Mir, L.M., Fernández, M.L. and Reigada, R. (2012) Effects of Dimethyl sulfoxide in Lipid Membranes Containing Cholesterol: A Comparative Study of Experiments in Silico and with Cells. PLoS ONE, 7, e41733.

https://doi.org/10.1371/journal.pone.0041733

[28] Haemmerich, D., Staelin, S.T., Tsai, J.Z., Tungjitkusolmun, S., Mahvi, D.M. and Webster, J.G. (2003) In Vivo Electrical Conductivity of Hepatic Tumors. Physiolog ical Measurement, 24, 251-260. https://doi.org/10.1088/0967-3334/24/2/302

[29] Stuchly, M.A. and Zhao, S. (1996) Magnetic Field-Induced Currents in the Human Body in Proximity of Power Lines. IEEE Transactions on Power Delivery, 11, 102-109. https://doi.org/10.1109/61.484005

[30] Haddar, D., Haacke, E.M., Sehgal, V., Delproposto, Z., Salamon, G., Seror, O. and Sellier, N. (2004) Magnetic Susceptibility Imaging: Theory and Applications. Journal of Radiology, 85, 1901-1908. https://doi.org/10.1016/S0221-0363(04)97759-1

[31] Yu, Z.W. and Quinn, P.J. (2000) The Effect of Dimethyl sulfoxide on the Structure and Phase Behavior of Palmitoleoylphosphatidylethanolamine. Biochimica et Biophysica Acta (BBA)-Biomembranes, 1509, 440-450. https://doi.org/10.1016/S0005-2736(00)00326-6

[32] Dorel, F. and Declerq, M. (1992) A Prototype for the Design-Oriented Symbolic Analysis of Analog Circuits. 1992 Proceedings of the IEEE Custom Integrated Circuits Conference, Boston, 3-6 May 1992, 12.5.1-12.5.4. https://doi.org/10.1109/CICC.1992.591292

[33] Antao, B.A.A. and El-Turky, F.M. (1992) Automatic Analog Model Generation for Behavioral Simulation. 1992 Proceedings of the IEEE Custom Integrated Circuits Conference, Boston, 3-6 May 1992, 12.2.1-12.2.4. https://doi.org/10.1109/CICC.1992.591289

[34] Kemper, U. and Mammen, H.T. (1994) Netlist and Behavioral Description of Macromodels for Analog Circuits. Conference on Modeling and Simulation, Barcelone, 1-3 June 1994, 979-984. 
[35] Koskinen, T. and Cheung, P.Y.K. (1992) Tolerance Analysis Using Behavioral Models. 1992 Proceedings of the IEEE Custom Integrated Circuits Conference, Boston, 3-6 May 1992, 3.4.1-3.4.4. https://doi.org/10.1109/CICC.1992.591101

[36] Yang, J., Huang, Y., Wang, X., Wang, X.B., Becker, F.F. and Gascoyne, P.R.C. (1999) Dielectric Properties of Human Leukocyte Subpopulations Determined by Electrorotation as a Cell Separation Criterion. Biophysical Journal, 76, 3307-3314. https://doi.org/10.1016/S0006-3495(99)77483-7

[37] Sacks, Z.S., Kingsland, D.M. and Lee, R. (1995) A Perfectly Matched Anisotropic Absorber for Use as an Absorbing Boundary Condition. IEEE Transactions on Antennas and Propagation, 43, 1460-1463. https://doi.org/10.1109/8.477075

[38] Raicu, V. (1999) Dielectric Dispersion of Biological Matter: Model Combining Debye-Type and Universal Responses. Physical Review E, 60, 4677-4680. https://doi.org/10.1103/PhysRevE.60.4677

[39] Wang, W. and Eisenberg, S.R. (1994) A Three-Dimensional Finite Element Method for Computing Magnetically Induced Currents in Tissues. IEEE Transactions on Magnetics, 30, 5015-5023. https://doi.org/10.1109/20.334289

[40] Steendijk, P., Mur, G., Van Der Velde, E.T. and Baan, J. (1993) The Four-Electrode Resistivity Technique in Anisotropic Media: Theoretical Analysis and Application on Myocardial Tissue in Vivo. IEEE Transactions on Biomedical Engineering, 40, 1138-1148. https://doi.org/10.1109/10.245632

[41] Mimi, M. (1990) An Investigation of Radiometer and Antenna Properties for Microwave Thermography. Doctoral Thesis, University of Glasgow, Glasgow. 